\title{
Effect of shed Configuration on AC Flashover Performance of Polluted Porcelain Insulators
}

\author{
Yawei $\mathrm{Li}^{1,}$, , Xinghai Zhang ${ }^{1, b}$, Xiaolei Yang ${ }^{2, \mathrm{c}}$, Huan Bai ${ }^{1, \mathrm{~d}}$ and Lei Sun ${ }^{3, e}$ \\ ${ }^{1}$ Electric Power Research Institute of State Grid Sichuan Electric Power Company, Chengdu, \\ China \\ ${ }^{2}$ State Grid Chengdu Power Supply Company, Chengdu, China \\ ${ }^{3}$ Graduate School at Shenzhen, Tsinghua University, Shenzhen, China

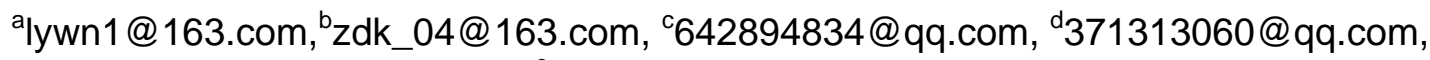 \\ eslsz_2014@126.com
}

\begin{abstract}
Keywords:Shed configuration, Porcelain insulator, pollution flashover, AC
Abstract.The flashover of polluted insulator is one of the serious problems which should be taken into account in the design and coordination of high voltage insulation. In order to clarify the influence of umbrella shape configurations on the pollution flashover characteristics of AC suspension porcelain insulators, pollution flashover tests have been operated for 11 types of insulators with different umbrella shape configurations. The results indicate that the specific leakage distance increase with the enlargement of diameter and height of insulators, and the increasing severities are relevant to their umbrella shapes. The specific leakage distance of normal type insulators increase more significantly than that of outer-rib insulators with geometrical dimensions enlarging. With equal diameter or height, the specific leakage distance of normal type insulator is bigger than that of outer-rib insulator. And the leakage distance availabilities of normal insulators are superior to that of outer-rib insulator with the same geometrical dimensions. The test results also demonstrate that the contaminated flashover performances of insulators with bigger geometrical dimensions are better than that with smaller geometrical dimensions with the same umbrella shape, this is because that the bigger the geometrical dimensions, the longer the leakage length, and the contamination flashover voltage of certain pollution level is determined by both the umbrella shape configuration and the leakage length of insulator.
\end{abstract}

\section{Introduction}

One of the most serious problems with power transmission by overhead lines is contaminated flashover [1]. Especially in recent years, threaten of contamination flashover become more intensity than before, due to the mass construction of UHV and EHV transmission lines and the aggravation of industrial pollution [2]. With the aim of reducing the harm of polluted flashover, it is necessary to optimize the design and selection of insulators. Therefore the impact of shape configurations on flashover voltage should be clarified.

Many researches have been carried out in order to investigate the relative between the umbrella shape configurations and the flashover characteristic of insulator. For cylindrical insulator, some papers state that the larger the diameter, the lower the flashover voltage [3-6], based on this conclusion, R. Matsuoka et al. further point out that the increase in diameter of a cylindrical insulator decrease the polluted deposit density on its surface, and the combined impact of both factors reduces the flashover voltage significantly [7]. M. Faselian et al. indiacated that with equal height under ribs, partial arcs prefer to choose the nearest path at the time of passing the entire leakage length for suspension porcelain insulator strings [8]. Therefore, it is preferable to have unequal under-ribs in order to improve the flashover voltage. That is the reason why under-rib type insulators with alternative long and short ribs are widespread used in transmission lines. Paper [9] declared that the specific leakage length increases with the diameter of insulator increasing for any profile of insulator.

All above researches about shape profiles are in regard to cylindrical or under-rib insulators, there is hardly any study about the influences of umbrella shape configurations on flashover voltage 
of outer-rib insulators. Therefore, 3 two outer-rib type, 2 three outer-rib type insulators were took into account, and 6 normal type insulators were chosen as references, 11 insulators in all were chosen for the tests of polluted flashover performances, and the impacts of diameter, height, leakage distance and modelling coefficient on polluted flashover performance were investigated.

\section{Artificial Pollution Test}

\subsection{Introduction of samples}

The parameters of 11 types of insulators are provided in table 1. As stated in table 1, the insulators can be classified as 3 types by umbrella shape, that is, normal type, two outer-rib type and three outer-rib type. The diagrams of the 3 types are provided as figure 1 .

Tab.1 parameters of insulators

\begin{tabular}{|c|c|c|c|c|}
\hline \multirow{2}{*}{ type } & $\begin{array}{c}\text { serial } \\
\text { number }\end{array}$ & $\begin{array}{c}\text { diameter } \\
\mathrm{mm}\end{array}$ & $\begin{array}{c}\text { height } \\
\mathrm{mm}\end{array}$ & $\begin{array}{c}\text { leakage } \\
\text { distance } \\
\mathrm{mm}\end{array}$ \\
\hline \multirow{4}{*}{ normal } & 1 & 255 & 146 & 295 \\
\cline { 2 - 5 } & 2 & 255 & 146 & 305 \\
\cline { 2 - 5 } & 3 & 280 & 170 & 332 \\
\cline { 2 - 5 } & 5 & 320 & 195 & 505 \\
\hline \multirow{3}{*}{$\begin{array}{c}\text { Two } \\
\text { outer- } \\
\text { rib }\end{array}$} & 7 & 340 & 205 & 550 \\
\cline { 2 - 5 } & 8 & 380 & 240 & 688 \\
\hline \multirow{2}{*}{$\begin{array}{c}\text { Three } \\
\text { outer- } \\
\text { rib }\end{array}$} & 10 & 430 & 146 & 400 \\
\cline { 2 - 5 } & 11 & 400 & 240 & 495 \\
\hline \multirow{2}{*}{ rib } & 9 & 390 & 205 & 560 \\
\hline
\end{tabular}

The normal type insulators have crowded under-ribs, and the height of ribs increase gradually from the pin to the edge of insulator. The outer-rib type insulators have open profiles, which make dust deposits on the surface of insulator harder than under-rib insulators.

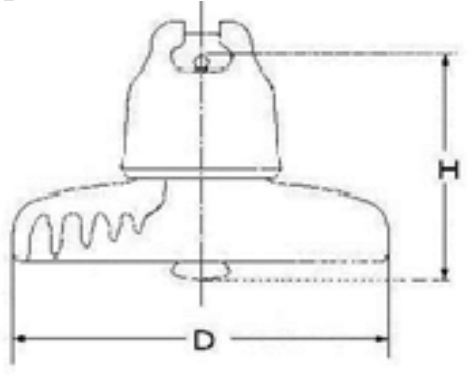

a. normal type

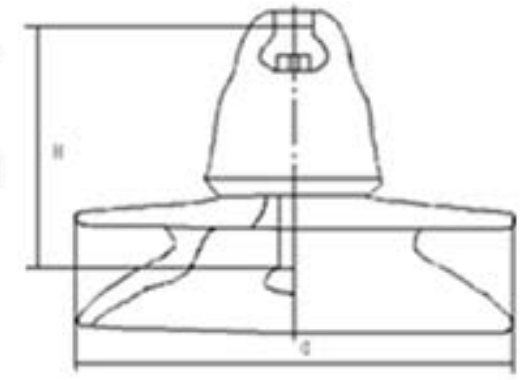

b. two outer-rib type

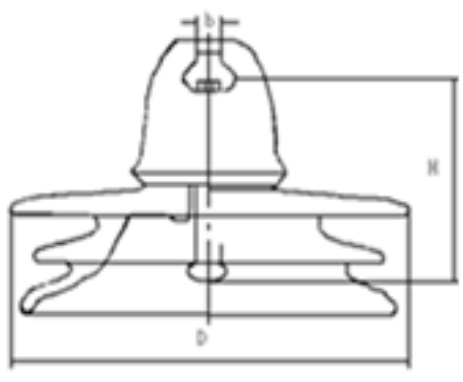

c. three outer-rib type

Fig.1 the AC suspension porcelain insulators

\subsection{Test method}

The tests were conducted in the fog chamber of EHVDC testing sites in China electric power research institute. The dimensions of fog chamber are $6 \mathrm{~m} \times 6 \mathrm{~m} \times 12 \mathrm{~m}$. The steam fog sprayed from the pip around the ground and near the wall of chamber, and the insulator strings can be humidified within $20 \mathrm{~min}$. The power source consists of a $200 \mathrm{kV} / 1000 \mathrm{kVA}$ high voltage transformer with a short-circuit impedance of lower than $0.47 \%$, and a protective resistance of $2 \mathrm{k} \Omega$. 


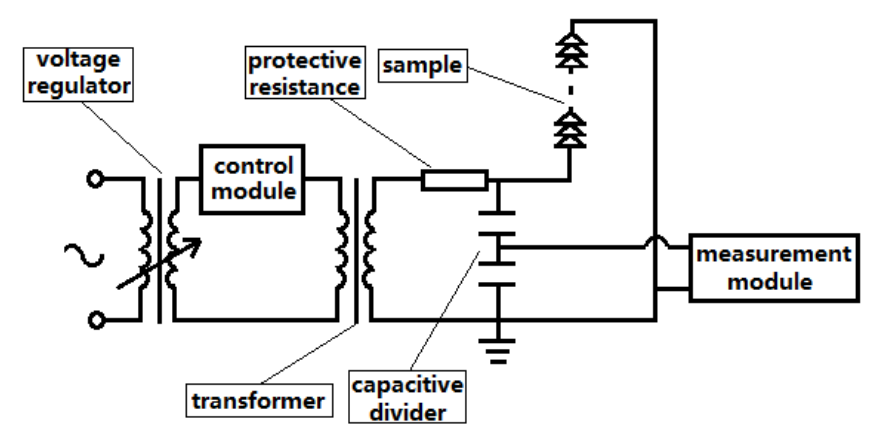

Fig. 2 schematic diagram of test circuit

Ration pasting method was adopted to simulate the artificial pollution, which consists of $\mathrm{NaCl}$ and kaolin. Up-and-down method was employed in this paper in order to determine the polluted flashover characteristics of insulators, and the test procedure $\mathrm{B}$ were selected according to ICE60507 [10].

\section{Analysis of Test Results}

The 50\% flashover voltage per unit of each type of insulator is provided in figure 3 . The test results indicate that the insulators with bigger geometric dimensions have better insulation performance than that of the insulators with smaller geometric dimensions.

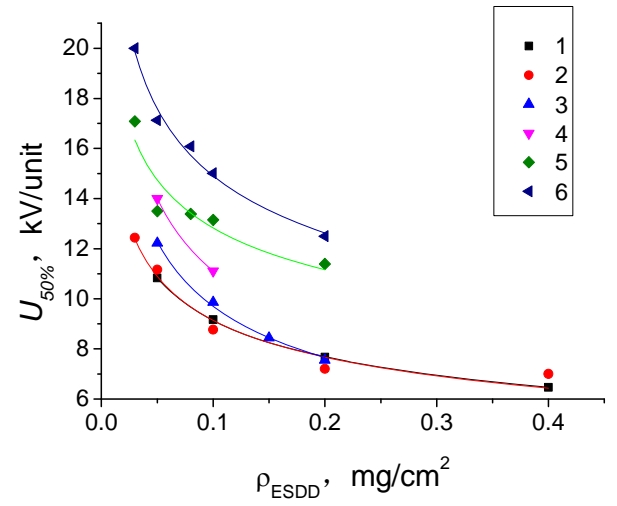

a. normal rib type insulators

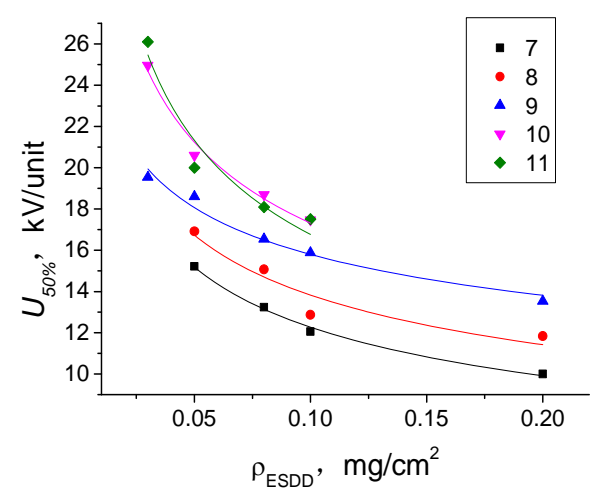

b. outer-rib type insulators

Fig. $3 U_{50 \%}$ per unit versus $\rho_{E S D D}$

The simulation curve of each insulator sample has also been provided in above figures. And the simulation formula is as equation (1):

$U_{50 \%}=A \rho_{E S D D}^{-n}$

$\rho_{E S D D}$ is the value of equivalent salt deposit density, $\mathrm{A}$ and $\mathrm{n}$ are constants and related to the shape of insulator.

\subsection{Effect of Diameter}

The influence of diameter $(D)$ on the pollution flashover voltage was mentioned in many papers. It is stated that the bigger the diameter, the lower the pollution flashover voltage with the other dimensions and test conditions are identical $[3,11]$. This is because that the increasing of diameter can enlarge the polluted area of insulator, resulting in the decrease of the contaminated layer resistance and the decrease of the flashover voltage [12]. It is clear that the specific leakage distance $\left(L_{f}\right)$ of AC suspension porcelain insulators increase with the increasing of diameter (figure 4), and the conclusion coincides with previous researches. Furthermore, the increasing severity of $L_{f}$ is influenced by the umbrella shape of insulator. It is clarified that $L_{f}$ of normal type insulators increasesmore rapidly than that of outer-rib type insulators. And the increasing tendency of $L_{f}$ caused by the increasing of diameter can be investigated with $\rho_{\mathrm{ESDD}}=0.05 \mathrm{mg} / \mathrm{cm}^{2}$ and $\rho_{\mathrm{ESDD}}=0.1 \mathrm{mg} / \mathrm{cm}^{2}$ (figure $4 \mathrm{a}, 4 \mathrm{~b}$ ). 


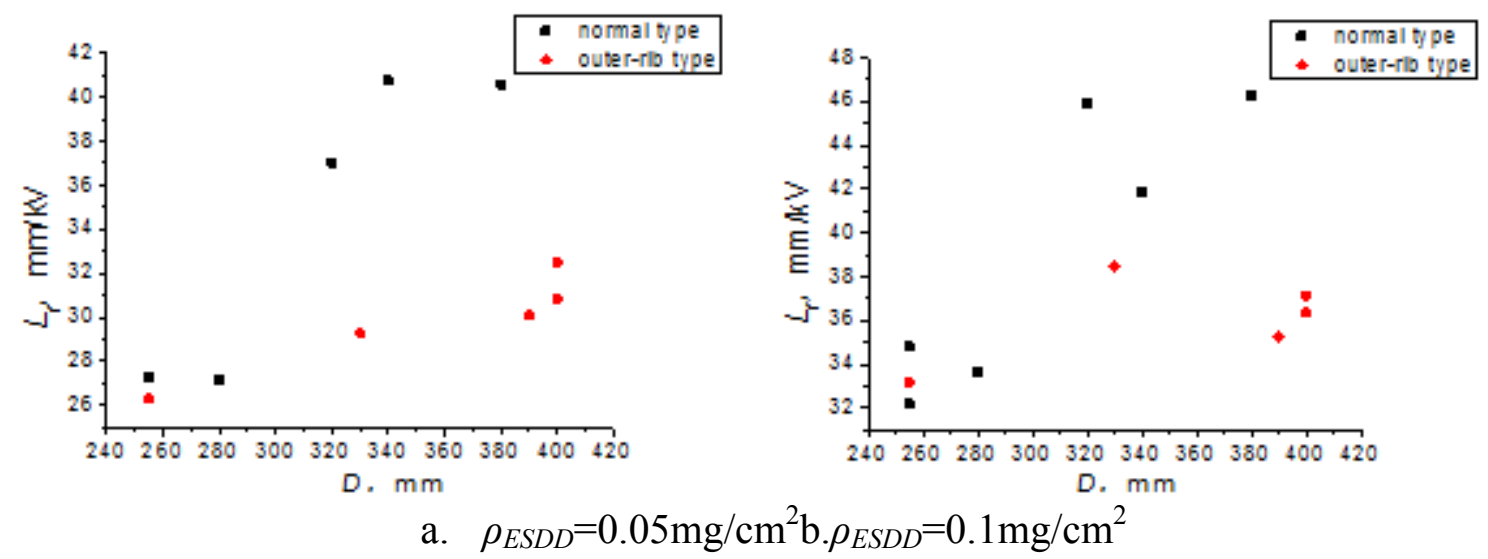

Fig. 4 diameter effect

\subsection{Effect of Height}

$L_{f}$ of normal type and outer-rib type insulators versus height $(H)$ is shown in figure 5 . It can be seen that $L_{f}$ of both normal type and outer-rib type insulators increase with height increasing. With the same height, $L_{f}$ of normal type insulators is bigger than that of outer-rib type insulators. The effect of $H$ on $L_{f}$ is similar with effect of $D$. With different $\rho_{E S D D}$ value, the changing of $L_{f}$ caused by the heightening of $H$ has the same tendency (figure 5a, 5b), and $L_{f}$ of normal type insulators increases faster than that of outer-rib type insulators. So the effect severity of $H$ on $L_{f}$ is also affected by the shape of insulator.
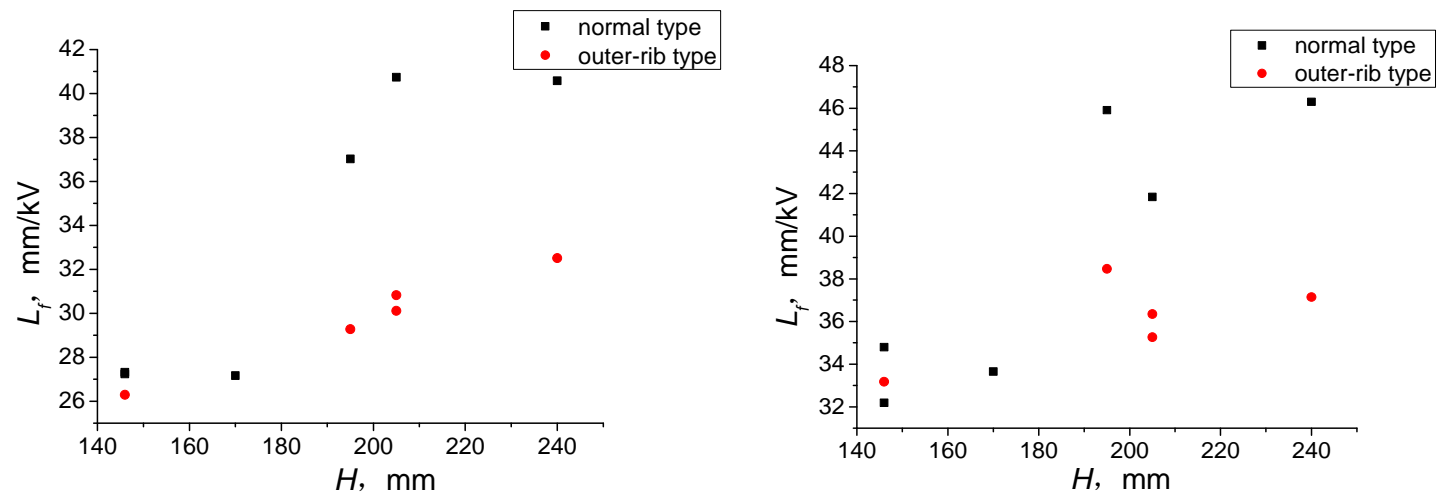

a. $\rho_{E S D D}=0.05 \mathrm{mg} / \mathrm{cm}^{2}$

Fig.5 height effect

b. $\rho_{E S D D}=0.1 \mathrm{mg} / \mathrm{cm}^{2}$

According to above analyses, it is cleared that $L_{f}$ increases with increasing diameter or height, and the increasing severities of $L_{f}$ for different umbrella shapes are different. The $L_{f}$ of normal type insulator increases faster than that of outer-rib type, which suggests that the influences of geometry dimensions on contaminated flashover performance are related with shape configurations of insulator. The height of under-ribs rise in turn from the pin to the edge of insulator, thus the partial arcs initiate from the pin can bridge the ribs easily, and then the efficiency of leakage distance reduces [8]. In turn, the partial arcs elongate close to the surface of outer-rib insulators, hence the efficiencies of leakage distance of these insulators are relatively higher than that of under-rib insulators. In conclusion, shape configurations affect the efficiency of leakage distance significantly.

\subsection{Compare of Per Unit U50\% of Different Insulators}

It has been observed that with increasing leakage distance, there is an increase in flashover voltage. According to table 1, the leakage distances of insulators increases with the increasing of leakage distance for each type of insulators. Figure 6 shows per unit $U_{50 \%}$ of insulators with different ESDD values $\left(0.05,0.1 \mathrm{mg} / \mathrm{cm}^{2}\right)$. It is clear that per unit $U_{50 \%}$ of insulators increase with the increasing of leakage distance. It can also be seen in figure 6 that the effective of leakage distance depends on the shape of the insulator to some extent, the effectives of leakage distance of outer-rib insulators are higher than that of normal type insulators.

The $U_{50 \%}$ of insulators with one or two similar geometry dimensions were compared in Figure 7 and 8 . 

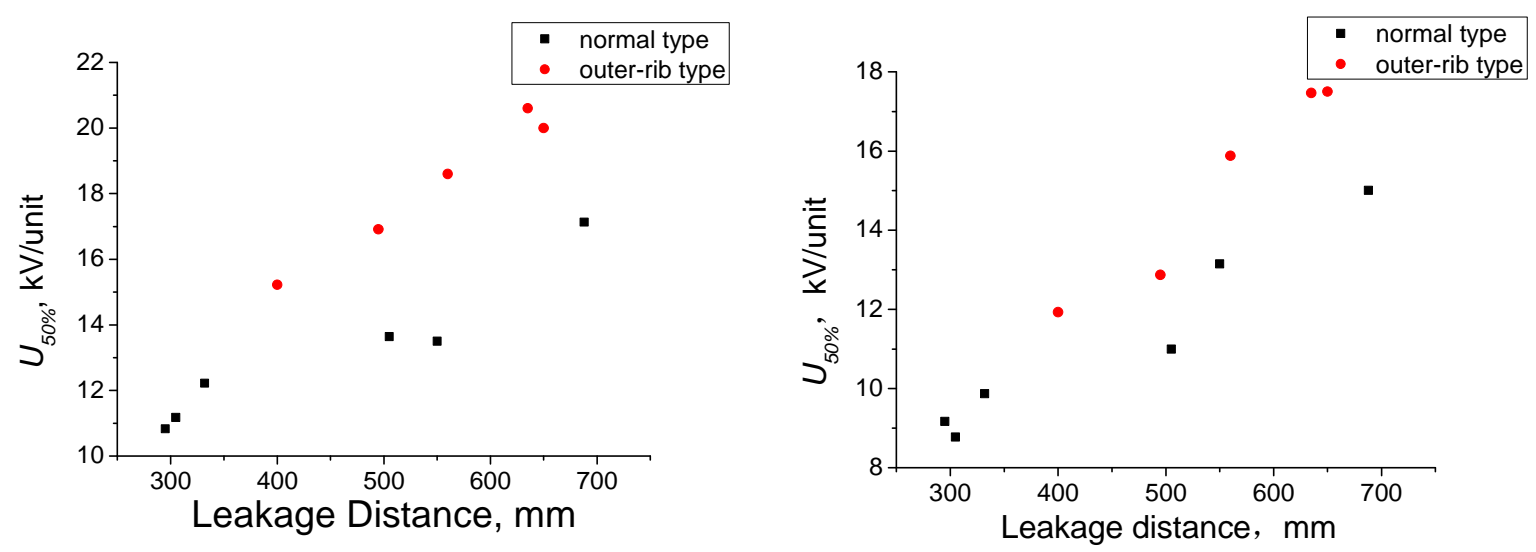

a. $\rho_{E S D D}=0.05 \mathrm{mg} / \mathrm{cm}^{2}$ b. $\rho_{E S D D}=0.1 \mathrm{mg} / \mathrm{cm}^{2}$

Fig.6leakage distance effect

The heightsof sample 5, 9 and 10 are similar, and their diameters increase in turn. It can be seen from figure 7 that the flashover voltage increases with the diameter increasing. The leakage distance increase with the increasing of diameter, on the other hand, the leakage distance utilization rate of outer-rib insulator is superior to that of under-rib insulator, so thepreferential sequence of flashover performance is 10,9 and 5 .

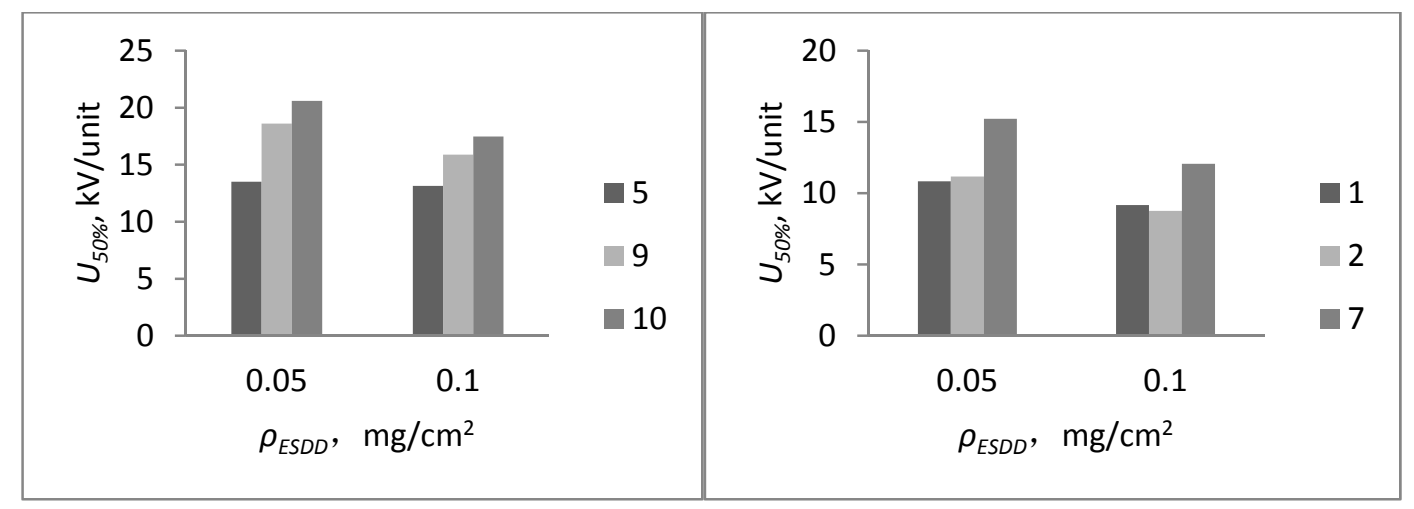

Fig. $7 U_{50 \%}$ per unit of insulators with similarheight (left)

Fig. $8 U_{50 \%}$ per unit of insulators with similar height and diameter (right)

Sample 1, 2 and 7 have similar height and diameter. Sample 1, 2 are normal type insulator; sample 7 is outer-rib insulator. According to figure 8, the two normal type insulators have approximate flashover voltages, and the outer-rib type insulator have the highest flashover voltage.

Figure 7, 8 proved that the outer-rib type insulators have the optimum pollution flashover performance compared to under-rib type insulators.

\subsection{Effect of Leakage Modelling Coefficient}

In order to review the total effect of diameter, height and leakage distance, modelling coefficient $F$ was brought in this paper. The definition of $F$ is as follows [2].

$F=D L / H^{2}$

$L$ is the leakage distance of insulator.

The contaminated flashover performance of each type of insulators versus modelling coefficient is provided in figure 9. It can be observed that the effect of modelling coefficient is intensively related to the type of insulator. 

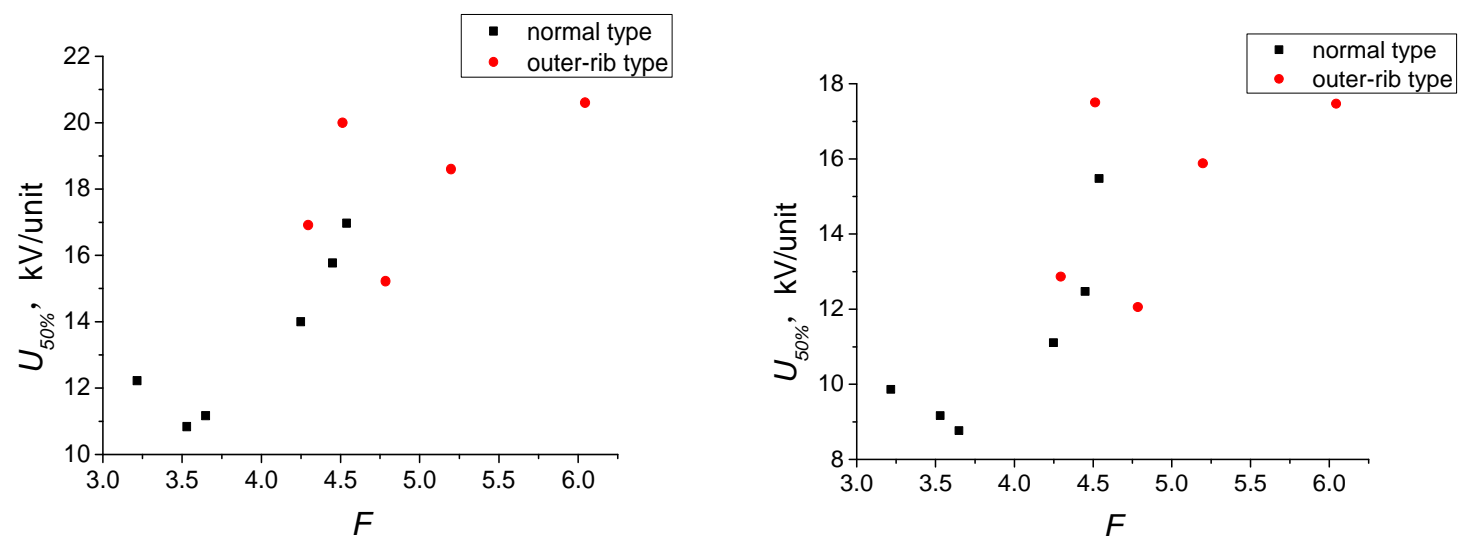

a. $\rho_{E S D D}=0.05 \mathrm{mg} / \mathrm{cm}^{2}$ b. $\rho_{E S D D}=0.1 \mathrm{mg} / \mathrm{cm}^{2}$

Fig.9 effect of modelling coefficient

The optimal values ranges of $F$ are discriminatory with different types of insulators according to the figures above. That should be attention in designing and selection of different types of insulators.

\section{Conclusions}

Aseries of tests have been operated in order to compare the contaminated flashover performance of 11 AC suspension porcelain insulators. The followings are concluded.

- The bigger the diameter and height, the larger the specific leakage distance of insulators with analogical shape. In other words, the increase of geometry dimensions will decrease theavailability of leakage distance.

- The severity of $L_{f}$ increasing arises from the enlargement of geometry dimensions is determined by the umbrella shape of insulator. With the increasing of insulator dimensions, $L_{f}$ of outer-rib insulators increases more slowly than that of normal type insulators.

- With the same ESDD, the polluted flashover performance of insulator is determined by both the availability of leakage distance and the leakage distance.

- In case of similar dimensions and pollution severity, $L_{f}$ of outer-rib type insulators is smaller than normal type insulators; and the flashover voltage of outer-rib insulators higher than that of normal type.

- The enlargement of modelling coefficient is helpful to the improvement of contaminated flashover performance.

\section{Reference}

[1] A. C. Baker, L. E. Zaffanella, L. D. Anzivino, et, al.,"A compariation of HVAC Contamination Performance of Station Post Insulators", IEEE Transactions on Power Delivery, Vol. 4, No. 2, April 1989.

[2] Guan zhicheng, Liu Yingyan, Zhou Yuanxiang, "Insulator and Outdoor insulation of PowerTransmission and Transformation Equipment”, Tsinghua University Press, Beijing, 2006.

[3] I. Kimoto, K. Kito and T. Takatori, “Anti-pollutionDesign Criteria for Line and Station Insulators”,IEEE Trans. PAS, Vol. 91, pp. 317-327, 1972.

[4] M. P. Verma, "Insulation Performance of DC Apparatus Housing under Pollution", ETZ Archiv,Vol. 5,pp. 269-275,1983.

[5] A. Pigini, D. Perin, F. Zagliani, N. Ramamoorty, C.S. Lakshminarasimha and V. B. Rammohan, "Performanceof Insulators for EHVDC Systems underPolluted Conditions", CIGRE Session Paper, No.33-11, 1988. 
[6] T. Kawamura, T. Seta, S. Tanabe and K. Naito,"Performance of Large Bushing Shells for EHVTransmission System under Polluted Conditions”, CIGRE Session Paper No. 33-03, 1988.

[7] R. Matsuoka, S. Ito, K. Sakanishi, "Flashover on Contaminated insulators with Different Diameters", IEEE Transactions on Electrical Insulation, Vol. 26, pp. 1140-1146, Dec. 1991.

[8] M. Faselian, C. Y. Wu, T. C. Cheng, H. I. Nourand L. J. Wang, "A Study on the Profile of HVDCInsulators: dc Flashover Performance”, IEEE Trans.EI, Vol. 24, No. 1, pp. 119-125, Feb 1989.

[9] R. Sundararajan, R. S. Gorur, "Effect of Insulator Profiles on DC Flashover Voltage under PollutedConditions"IEEE Transactions on Dielectrics and Electrical Insulation Vol. 1 No. 1, pp. 124-132, February, 1004.

[10]IEC 60507: 1991. Artificial pollution tests on high-voltage insulators to be used on a.c. systems.

[11]I. Kimoto, T. Fujimura, and K. Naito,"Performance of Insulators for Direct Current Transmission Line under Polluted Condition”, IEEE Trans. on PAS, Vol. 92, pp. 943-949, May/Jun 1973.

[12]P. J. Lambeth, "Effect of Pollution on HV Outdoor Insulators", Proc. IEE, Vol. 118, no. 9R, pp. 1107-1130, Sep 1971. 